\title{
Design, Implementation and Simulation of Non-Intrusive Sensor for On-Line Condition Monitoring of MV Electrical Components
}

\author{
Muhammad Shafiq1, Matti Lehtonen', Lauri Kutt'1, Muzamir Isa ${ }^{2}$ \\ ${ }^{1}$ Department of Electrical Engineering, School of Science and Technology, Aalto University, Espoo, Finland \\ ${ }^{2}$ School of Electrical Systems Engineering, Universiti Malaysia Perlis, Malaysia \\ Email: muhammad.shafiq@aalto.fi, matti.lehtonen@aalto.fi, lauri.kutt@aalto.fi, Smuzamir@unimap.edu.my
}

Received 6 August 2014; revised 29 August 2014; accepted 16 September 2014

Copyright (C) 2014 by authors and Scientific Research Publishing Inc.

This work is licensed under the Creative Commons Attribution International License (CC BY).

http://creativecommons.org/licenses/by/4.0/

(c) (i) Open Access

\begin{abstract}
Non-intrusive measurement technology is of great interest for the electrical utilities in order to avoid an interruption in the normal operation of the supply network during diagnostics measurements and inspections. Inductively coupled electromagnetic sensing provides a possibility of non-intrusive measurements for online condition monitoring of the electrical components in a Medium Voltage (MV) distribution network. This is accomplished by employing Partial Discharge (PD) activity monitoring, one of the successful methods to assess the working condition of MV components but often requires specialized equipment for carrying out the measurements. In this paper, Rogowski coil sensor is presented as a robust solution for non-intrusive measurements of PD signals. A high frequency prototype of Rogowski coil is designed in the laboratory. Step-by-step approach of constructing the sensor system is presented and performance of its components (coil head, damping component, integrator and data acquisition system) is evaluated using practical and simulated environments. Alternative Transient Program-Electromagnetic Transient Program (ATP-EMTP) is used to analyze the designed model of the Rogowski coil. Real and simulated models of the coil are used to investigate the behavior of Rogowski coil sensor at its different stages of development from a transducer coil to a complete measuring device. Both models are compared to evaluate their accuracy for PD applications. Due to simple design, flexible hardware, and low cost of Rogowski coil, it can be considered as an efficient current measuring device for integrated monitoring applications where a large number of sensors are required to develop an automated online condition monitoring system for a distribution network.
\end{abstract}

\section{Keywords}

Non-Intrusive, Sensors, Condition Monitoring, Partial Discharge, Rogowski Coil, ATP-EMTP 


\section{Introduction}

The major deriving forces to modernize the current power grid include increasing requirements for reliability, efficiency and safety of power grid along with optimization of capital assets while minimizing the operation and maintenance cost. Some of the greatest effects on reliability of a power distribution network are provided by various types of failures due to environmental and operational stresses [1]. Insulation degradation is one of the most frequent causes of the failure in the critical and expensive power components such as, motors, generators, transformers, switchgear, and power lines. One of the methods to predict the incoming insulation faults is to perform on-line condition monitoring of the network components. Partial Discharge (PD) diagnostic is a wellknown technique for insulation condition monitoring. PD is the process of localized dielectric breakdown of a small portion (cavities, voids, cracks or inclusions) of a solid or liquid electrical insulation part which is under high voltage stress during operation [2]. The electrical stress due to applied voltage causes discharges within the defective portion of the insulation. There are different phenomena which appear during discharges and give rise to respective detection indicators such as electromagnetic radiation, sound or noise, thermal radiation, gas pressure, chemical formation, and electromagnetic impulses [3]-[6]. The PD measurement sensor technology is based on the type of the energy exchange which takes place during above mentioned discharge phenomena. In this paper electromagnetic impulses are considered for assessment of the PD activity.

Due to rapid displacement of the charges during discharge event, voltage and current transient appears in the form of electromagnetic waves. These signals travel away from the site of origin along the power lines and can be measured by resistive, capacitive or inductive methods [7]. A variety of sensors are available for measurement of electromagnetic signals. However, considering practical aspects such as low cost, high bandwidth, good sensitivity, saturation characteristics, high linearity, and wide operating temperature, Rogowski coil has been regarded as a favorite tool for high-frequency current-sensing purposes [7]. Its flexible design provides the possibility to install it in a variety of physical locations especially in the tight spaces that may be inaccessible with typical iron-core current transformers. For MV overhead covered conductor lines, it can directly be installed around the CC line. For an MV cable network and cable accessories (joints and terminations), switchgears, and transformers, the coil can easily be installed around the earth straps to detect the fault current. Moreover it can also be used for normal current measurements at the substation. Researchers and engineers have been using Rogowski coil for high-amplitude sinusoidal currents and transients as well as low-amplitude and high-frequency signals in power systems and power electronics applications [8]-[11]. Nowadays it is also being used for detection and localization of insulation and short circuit faults while its application for relay protection is impressive as well [12]. Obtaining simplicity in the design, better accuracy for a wide range of amplitude and frequency of measured signal, and low cost are the challenges during development of a sensor.

In this paper, the main features, design and construction features of Rogowski coil have been explored. Rogowski coil sensor has been identified as a composite of four essential and sequential components: Rogowski coil head, damping component, integrator and Data Acquisition System (DAS). Step-by-step implementation of Rogowski coil is described and performance of each stage is evaluated for low-amplitude and high-frequency transient current signals. The operation of the Rogowski coil is simulated in ATP-EMTP simulation software environment which provides an in-depth analysis, the verification of the coil prototype and the possibility of developing further PD diagnostic techniques for distribution lines.

\section{Components of Rogowski Coil Sensor}

A measuring sensor transacts a complete measuring function from initial detection to final indication of the measured quantity as described in Figure 1. Considering Rogowski coil sensor, the initial detection is done by a current sensing coil which acts as an interface between primary and measured current. Intermediate signal

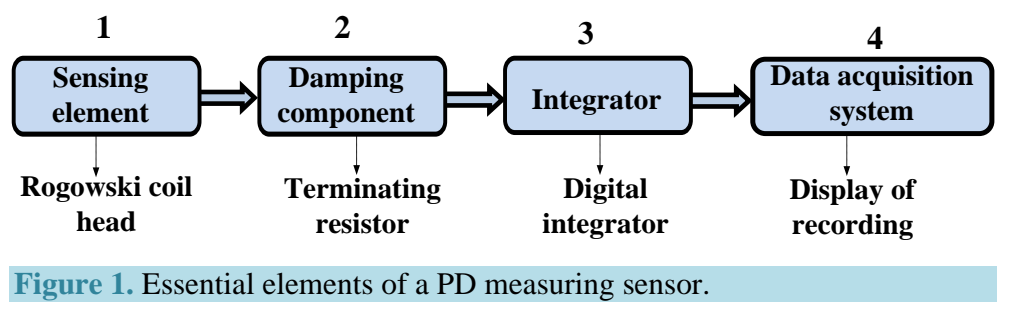


processing consists of signal conditioning based on the operational features of the coil, its physical and electrical characteristics, and the purpose (requirement) of measurements and diagnostics. This is accomplished by damping component and integrator. Final indication is displayed or recorded with the help of a suitable Data Acquisition System (DAS). This recorded data can be transported to personal computers to be used for further investigations. Figure 1 represents the sequential construction and operation of Rogowski coil measuring sensor while its implementation is depicted in Figure 2. The detailed description and implementation of each component is explained further in this section.

\subsection{Coil Head}

The Rogowski coil's main sensing part is composed of toroidal wound coil with $n$ number of turns, on an air-core (dielectric) former of constant cross-sectional area. The air-core is split in one location to allow assembly of the coil head unit. As shown in Figure 3, the wire is wound such that the winding start from the first end, progresses towards the other end and returning through the centre of the coil back to the first end, so that both terminals are at the same end of the coil. Number of turns $(n)$, outer diameter of coil $\left(d_{o}\right)$, and inner diameter of coil $\left(d_{i}\right)$ are the major physical parameters of coil head (see Table 1$)$. Its physical parameters are selected based on the application requirements of the coil. Space for installation, bandwidth, and sensitivity of the coil are the main concerns required to initiate the design.

\subsection{Damping Component}

Every piece of wire has resistance and inductance while every two wires present some capacitance during an electrical application [13]. Response of the Rogowski coil to primary current signal can be well explained by developing electrical model of this electromagnetic device. Lumped parameter model is used in this work where $R_{c}, L_{c}$ and $C_{c}$ are structure-based inherent self-resistance, self-inductance and self-capacitance of the Rogowski coil. During current measuring operation, the sensed signal passes through RLC circuit of the coil which

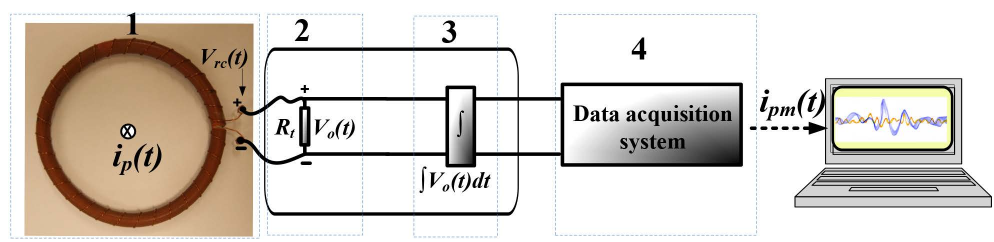

Figure 2. Stages of constructions of Rogowski coil measuring sensor.

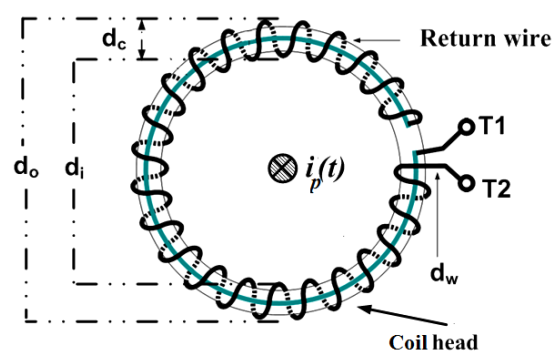

Figure 3. Physical model of the Rogowski coil.

Table 1. Physical parameters of Rogowski coil head.

\begin{tabular}{ccc}
\hline Name of the parameter & Symbol & Specification \\
\hline Number of turns & $n$ & 30 \\
Outer diameter of coil & $d_{o}$ & $16.1 \mathrm{~cm}$ \\
Inner diameter of coil & $d_{i}$ & $14.1 \mathrm{~cm}$ \\
Core diameter & $d_{r c}$ & $1.96 \mathrm{~cm}$ \\
\hline
\end{tabular}


introduces oscillations in the output voltage and current. The rate of these oscillations is determined by the resonant frequency of the coil. Such oscillations can be damped by properly terminating the response of the coil with a suitable resistance which is normally connected across the output terminal of the coil.

\subsection{Integrator}

The output voltage of Rogowski coil which is induced by the variable current passing through the primary conductor is proportional to the derivative of primary current. The main operating principle is thus described with Farady's law. Due to very nature of the electrical circuit of Rogowski coil, oscillations are introduced due to second order equivalent circuit characteristics. These factors affect the output of the Rogowski coil sensor (with reference to primary signal). Reliability of the measurement depends on how accurately the primary signal is captured. In order to recreate the original waveform of the measured signal, first step was to remove sensor's oscillations using damping component (described above). An integration operation is needed to process differential output to provide output waveform (measurement result) to match the measured primary current waveform.

Rogowski coil is an air-core induction sensor and relatively low number of turns are required to guarantee the high-frequency operation. Therefore the current transfer ratio (output current to input current) is very low; even minor resistive losses can significantly reduce the amplitude of the measured signal in the sensor's output. The specific calibration of the sensor transfer rate can thus be required to achieve the accurate amplitude of the measured signal.

\subsection{Data Acquisition System}

The output of Rogowski coil sensor is an analogue signal. For more detailed analysis and recording of the measured transients patterns, the signal is passed on to a DAS system and digitized. This incorporates in the first stage an Analog-to-Digital Converter (ADC) with very high sampling rate, in order to capture the extremely short-time transients of the PDs occurring on the power line. The Nyquist criterion states that the sampling frequency of a system needs to be at least twice of the frequency needed to be captured. The bandwidth needed to capture the PD traces would reach several tens $\mathrm{MHz}$, thus the sampling frequency required from the DAS would be in tens of millions of samples per second (MS/s) also. The ADC would need to have at least 8-bit resolution (better higher), therefore having multiple sensors in a substation would mean very high data transfer bandwidth.

The second stage of the DAS would be initial processing of the results to provide reduction of the data to be processed. The PD transients have specific waveforms and recognition/detection of such waveforms can be then used for triggering the further storage and processing operations. As a result of such event filtering, the data stored will be limited in a window of some thousands of samples at each detected PD occurrence, or even less. The data bandwidth is reduced for easy data storage or transmission to data centers for further analysis. Suitable equipment for establishing the DAS would be for example Digital Storage Oscilloscopes (DSO) in the laboratory measurements or on-chip data logger systems can be used for on-site applications.

\section{Stage-Wise Design and Implementation of Components of Rogowski Coil Sensor}

\subsection{Physical and Electrical Model of the Coil Head}

When the coil is placed around the conductor carrying alternating or transient current $i_{p}(t)$ to be measured (see Figure 2), the voltage $V_{r c}(t)$ induced within the coil is expressed as

$$
V_{r c}(t)=-M_{c} \frac{\mathrm{d} i_{p}(t)}{\mathrm{d} t}
$$

where $M_{c}$ is mutual inductance or sensitivity of the coil, expressed in $V / A$ at a specific frequency. Mutual Inductance depends on the number of turns of the coil, cross sectional area of the core, and the diameter of the toroid (to determine the radial distance of the coil winding from the current carrying conductor placed at the center of the coil). The physical parameters of the Rogowski coil head are given in Table 1 . The value of electrical parameter depends on the physical design of the coil. Table 2 represents the measured electrical parameters of the Rogowski coil head. The details of the parameters measuring methodology has been described by the authors in [14]. The lumped parameters (RLC) equivalent electrical model is shown in Figure 4. 
Table 2. Electrical parameters of Rogowski coil head.

\begin{tabular}{ccc}
\hline Name of the parameter & Symbol & Specification \\
\hline Self-resistance of coil & $R_{c}$ & $0.71 \Omega$ \\
Inductance of coil system & $L_{c}$ & $1.19 \mu \mathrm{H}$ \\
Capacitance of coil system & $C_{c}$ & $14.7 \mathrm{pF}$ \\
Measuring resistance & $R_{m}$ & $1 \mathrm{M} \Omega$ \\
\hline
\end{tabular}

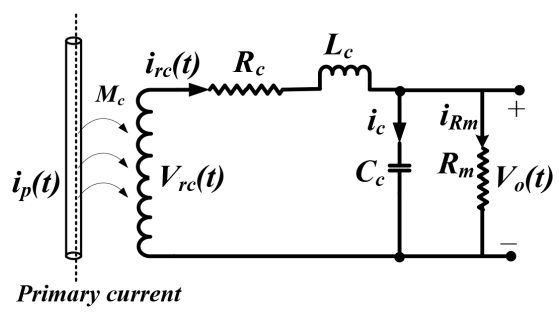

Figure 4. Electrical model of the Rogowski coil head.

Equation (1) presented the basic operation of a Rogowski coil by providing the induced voltage $V_{r c}(t)$ within the coil due to primary signal $i_{p}(t)$. The voltage collected at the output terminals of the coil is $V_{o}(t)$ as shown in Figure 4 includes also effects of self-inductance $L_{c}$, self-capacitance $C_{c}$ and active input resistance (the DSO has $R_{m}=1 \mathrm{M} \Omega$ ). It has be pointed out that the Rogowski coil sensor system includes the capacitance of the connection cables and the active input capacitance, which have been counted for in the total $C_{c}$ value. At lower frequency range, the parameters $L_{c}$ and $C_{c}$ of the coil do not have considerable effect and therefore $V_{o}(t) \cong V_{r c}(t)$. However at higher frequency range $V_{o}(t)$ can be expressed by the transfer function as

$$
V_{o}(t) \cong V_{r c}(t) V_{o}(s)=\frac{\frac{1}{L_{c} C_{c}}}{s^{2}+\frac{1}{L_{c} C_{c}}\left(\frac{L_{c}}{R_{m}}+R_{c} C_{c}\right) s+\frac{1}{L_{c} C_{c}}\left(\frac{R_{c}}{R_{m}}+1\right)} V_{r c}(s)
$$

During high frequency measurements, the interaction of sensed signal with RLC parameters results in significant energy exchange between the coil's self-inductance and self-capacitance, and causes oscillations with a resonant frequency $\omega_{c}$ which can be calculated as

$$
\omega_{c}=\sqrt{\frac{1}{L_{c} C_{c}}}=37.6 \mathrm{MHz}
$$

To ensure the practical response of Rogowski coil, a laboratory test is made. A PD pulse is injected from a PD calibrator into a simple test circuit as shown in Figure 5. The primary current pulse in the test line is measured by a commercial High Frequency Current Transformer (HFCT) and is shown in red color in Figure 6(a). This current pulse will be used as reference primary signal further in this paper. The captured output voltage of Rogowski coil is shown as time-domain plot in blue color in Figure 6(a). This oscillating signal is the measured signal $V_{o}(t)$ which needs to be processed during the incoming stages of the sensor development in order to get the primary signal $i_{p}(t)$. Fast Fourier Transform (FFT) of the captured response shown in Figure 6(b) represents the frequency response of the coil's output voltage. The resonant frequency of measured response is determined as 37.6 MHz. The resonant frequency reflects the value of the LC parameters of an induction sensor. Therefore, exact match of calculated and practically measured resonant frequency validates the accuracy of the measured electrical parameters.

\subsection{Coil Head Along with Damping Component}

In the previous, the output voltage $V_{o}(t)$ was measured by the DSO in the presence of a very large active 


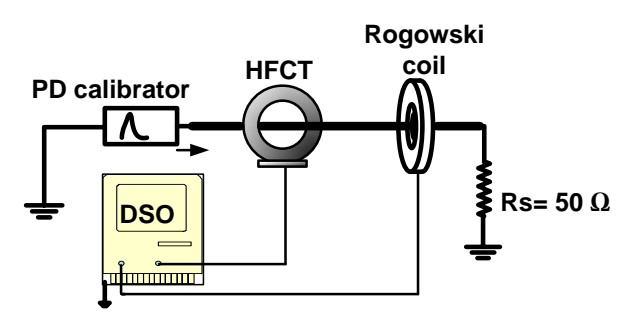

(a)

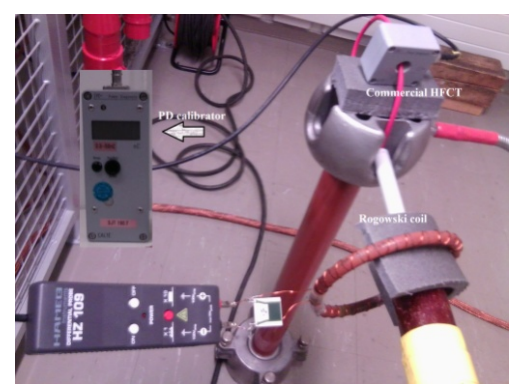

(b)

Figure 5. Experimental setup for measurement of PD current pulse. (a) Circuit model; and (b) Laboratory implementation.

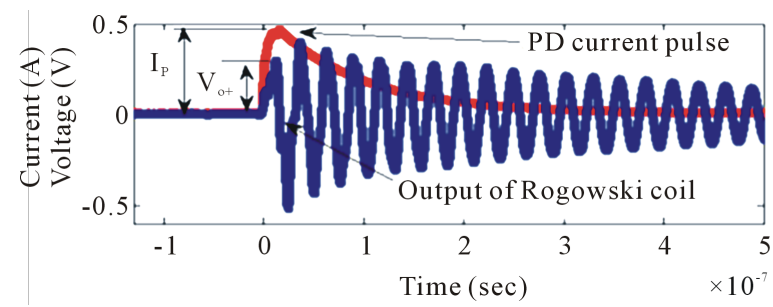

(a)

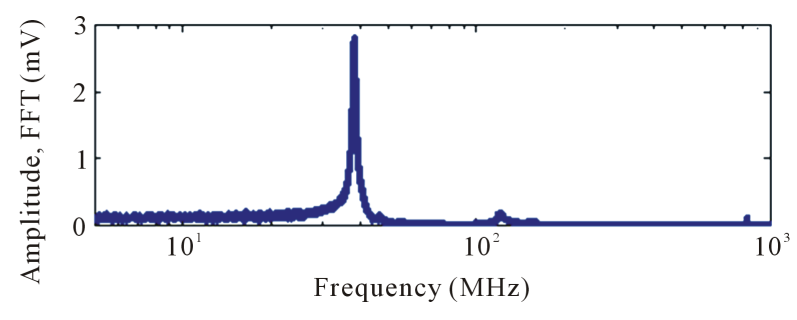

(b)

Figure 6. Measured response of Rogowski coil. (a) Time domain plot; (b) Frequency domain plot.

measurement device input resistance $R_{m}$. With this measuring setup, Rogowski coil operates in an un-damped mode. In order to damp the oscillation, a suitable value of terminating resistance is required which should be a specific ratio of the characteristics impedance of the Rogowski coil as shown in Figure 7. Based on the terminal loading, the Rogowski coil can operate in over damped, undamped, and critically damped modes, as shown in Figure 8. Suitability of the operating mode depends upon the measured waveform of the output which aims at to be the waveform of the primary signal.

The output voltage can be expressed as

$$
V_{o}(t)=V_{r c}(t)+V_{r c}(t) \mathrm{e}^{-\xi \omega_{C} t} \sin \left(\omega_{c} \sqrt{1-\xi^{2}} t\right)
$$

The response $V_{o}(t)$ of the coil can be divided into two parts:

1) $V_{r c}(t)$, the forced response due to primary signal and;

2) $V_{r c}(t) \mathrm{e}^{-\xi \omega_{C} t} \sin \left(\omega_{c} \sqrt{1-\xi^{2}} t\right)$, the natural response due to sensor's operation.

where $\xi$ is the damping coefficient. Natural response reflects coil's circuit properties which hides the information of the original measured signal. As visible from Equation (4), damping coefficient can be efficiently used to control the effect of oscillations in the output voltage of Rogowski coil. Considering Equation (2), the classical $2^{\text {nd }}$ order behavior of the coil's electrical model can be represented by the characteristic equation written as

$$
s^{2}+2 \xi \omega_{c} s+\omega_{c}^{2}=0
$$

Comparing with the transfer function, the damping coefficient $\xi$ for coil model without damping component can be an be expressed as

$$
\xi=\frac{1}{2 \cdot \omega_{c} L_{c} C_{c}}\left(\frac{L_{c}}{R_{m}}+R_{c} C_{c}\right)
$$

whereas the damping coefficient with terminating resistance $\xi_{t}$ can be written as

$$
\xi_{t}=\frac{1}{2 \cdot \omega_{c} L_{c} C_{c}}\left(\frac{L_{c}}{\left(R_{t} \| R_{m}\right)}+R_{c} C_{c}\right) .
$$




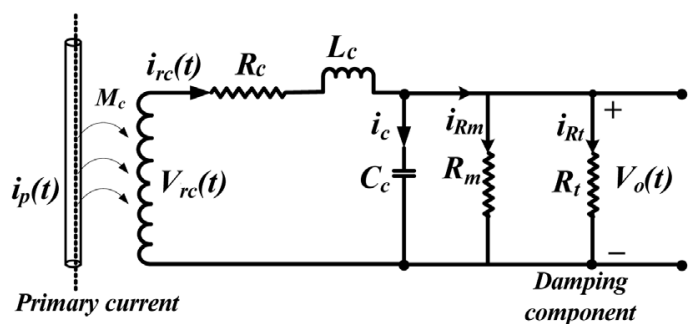

Figure 7. Electrical model of the Rogowski coil.

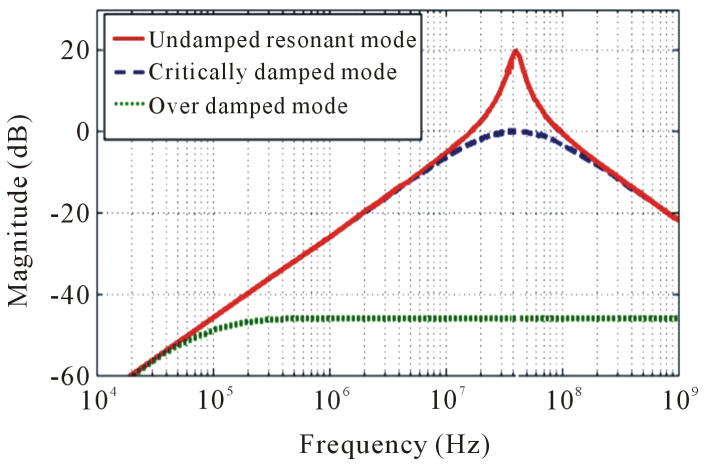

Figure 8. Modes of Rogowski coil operation based on the value of damping.

Here it should be noted that damping coefficient depends on all the four parameters ( $L_{c}, C_{c}, R_{c}$ and $R_{m}$ ) of the coil. The parameters $L_{c}, C_{c}$ and $R_{c}$ are fixed for a certain physical design of Rogowski coil. Therefore one of the efficient ways to specify value of $\xi$ is by variation in the $R_{m}$.

The coil operation properties in different modes (for different values of terminating resistance) can be described in the frequency plot as shown in Figure 8. The peak transfer value at $37.6 \mathrm{MHz}$ for undamped operation indicates the presence of high amplitude of oscillation. The amplitude of oscillations is reduced for the critically damped mode while the magnitude transfer rate has been considerably reduced for overdamped mode of operation, not only for the oscillations at single resonant point. The reduction or eliminations of oscillations can also be visualized in the poles zero plot for the designed coil. The solution of (4) results in a pair of poles as

$$
\left.\begin{array}{l}
P_{1}=-\xi \omega_{c}+\omega_{c} \sqrt{\xi^{2}-1} \\
P_{2}=-\xi \omega_{c}-\omega_{c} \sqrt{\xi^{2}-1}
\end{array}\right\} .
$$

For different values of terminating resistance, the location of poles provides a quantitative view of presence of oscillations within the output of Rogowski coil as shown in Figure 9. Location of poles from the real axis quantifies the presence of oscillations. It can be seen that for $R_{t}>Z_{c}$ the poles are away from real axis showing higher oscillations. It has been identified in [15] that for

$$
R_{t}=\frac{1}{2 \omega_{n} C_{c}}=\frac{Z_{c}}{2}
$$

Here $Z_{c}$ is the characteristic impedance of the coil. Using such damping component value, the poles still lie on real axis, while their magnitude has the highest value. The poles can have even higher magnitude values, however in such case the poles will also have an imaginary part. The removal of the oscillations from the output is beneficial for the sensor application, as removal of natural oscillatory response modifies (4) as

$$
V_{o}(t) \cong V_{r c}(t)=-M_{c} \frac{\mathrm{d} i_{p}(t)}{\mathrm{d} t} .
$$

Figure 10 shows the transformation of the signal from the output of stage 1 to the output of stage 2 . The compensation of the effect of time differential and mutual inductance can be carried out by integration and calibration. 


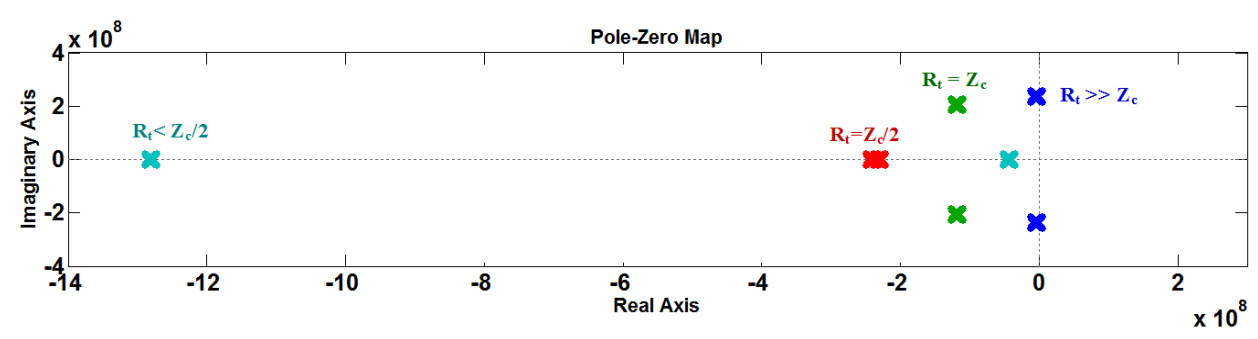

Figure 9. Effect of damping on the location of poles for different value of damping resistance.

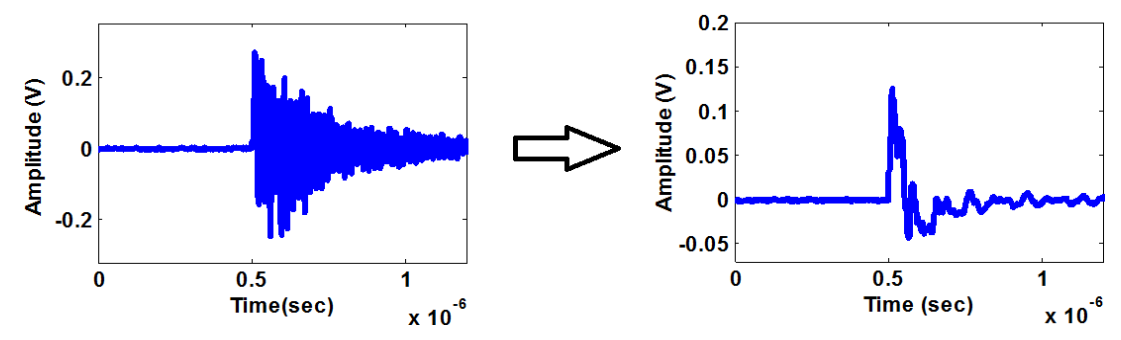

Figure 10. Signal conditioning from (a) undammped mode to (b) critically damped mode.

\subsection{Coil Head Along with Damping Component and Integrator}

Integration of the output of Rogowski coil can be performed by one of two common means, (1) by use of an electrical or electronic integrator, or (2) by using numerical integration in software after the coil output voltage is digitized [16]. In order to avoid complex circuit and expensive components, the numerical integration is proposed to be done by the built-in numerical functions of the DSO. The sampling frequency is selected based on the resonant frequency (considering Nyquist criterion) of the Rogowski coil. The digital integration can be expressed as

$$
i(N)=i(N-1)+\frac{1}{2 f_{s}}[V(N-1)+V(N)] \quad N>0,
$$

where $i$ is the current obtained by the digital integration of a voltage signal $V, f_{s}$ is sampling frequency and $N$ is the order number of sample. The digital integration recreates the current as

$$
i_{m}(t)=\int V_{o}(t) \mathrm{d} t
$$

The amplitude of the induced voltage is reduced by a factor $M_{c}$. Similarly resistive or stray losses can further reduce the amplitude of the measured pulse. Calibration of the measured signal can be done by comparing the measured signal $i_{m}(t)$ of the Rogowski coil with the reference signal. The calibration factor $K_{\text {cal }}$ can be calculated as

$$
\frac{i_{p}(t)}{i_{m}(t)}=K_{\text {cal }}
$$

where $K_{\text {cal }}$ depends on the $M_{c}$ and the stray losses $K_{\text {st }}$. Thus the $i_{p m}(t)$ can be obtained as

$$
i_{p m}(t)=i_{m}(t) K_{\text {cal }}
$$

The overall measurement scenario of the primary current $i_{p}(t)$ processed by the described stages of sensor to provide $i_{p m}(t)$ as the final outcome is shown in Figure 11 and Figure 12.

\subsection{Data Capturing Using Suitable Data Acquisition System}

PD transients are high frequency signals therefore a high sampling rate is required to capture the signals reliably without losing any information. During laboratory measurements, a sampling rate of $2.5 \mathrm{GS} / \mathrm{s}$ is used with the help of DSO. However it is important to take into factor the factor of sampling rate because it affects the economical parameters in terms of cost of high frequency processors and large memory for data storage. For real 


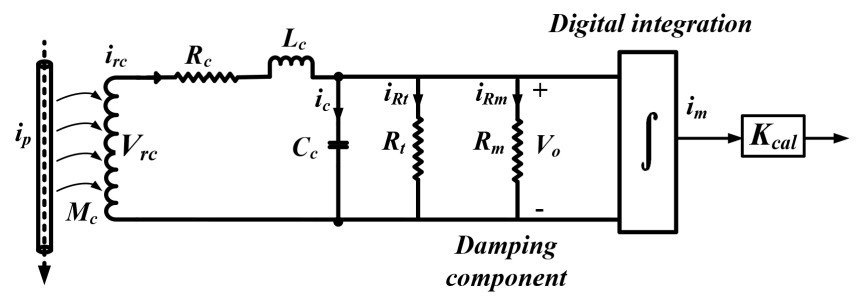

Figure 11. Complete electrical equivalent circuit of Rogowski coil measuring sensor.

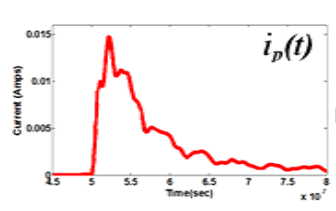

(a)

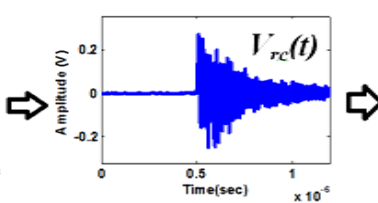

(b)

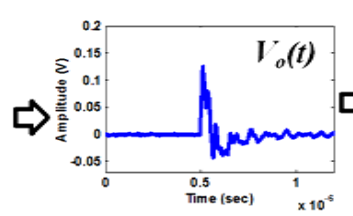

(c)

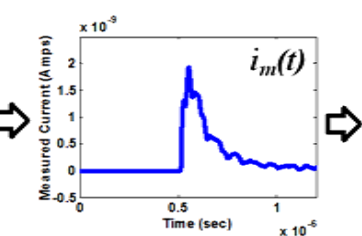

(d)

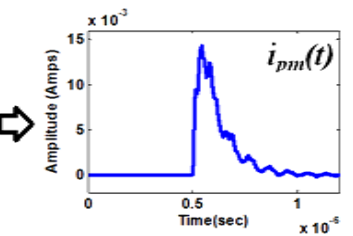

(e)

Figure 12. Signal wave-shapes at different stages of sensor design. (a) Primary current pulse $i_{p}(t)$ to be measured; (b) Signal sensed by coil head; (c) Damped signal; (d) Integrated signal and (e) Calibrated signal as final outcome primary measured signal $i_{p m}(t)$.

applications, such high sampling rate $(2.5 \mathrm{GS} / \mathrm{s})$ is not necessary. A suitable range of required sampling rate can be estimated by the resonant frequency of the measuring sensor. The resonant frequency of the Rogowski coil used in this work is $37.6 \mathrm{MHz}$. Therefore, considering Nyquist criterion, $76 \mathrm{MHz}$ or more, is a suitable sampling frequency for capturing the PD data.

\section{Simulation of Rogowski Coil Sensor Using ATP-EMTP}

Rogwoski coil is simulated using ATP-EMTP power system transients' simulation software. In literature, the pulse used in different simulation software is mostly based on the waveforms characterized by its mathematical model described as [13]

$$
i_{\text {pulse }}(t)=A\left(\mathrm{e}^{-\alpha_{1} t}-\mathrm{e}^{-\alpha_{2} t}\right)
$$

where $A$ is the peak value of the pulse, $\alpha_{1} t$ is the rise time and $\alpha_{2} t$ is half of the fall time. In this work, the data of practically captured PD current pulse is imported in ATP to be measured by the simulated model of Rogowski coil. Both mathematical and practical current pulses are shown in Figure 13(a). It can be clearly seen that practical current pulse contains fast variations during rising and fall slopes of the pulse. This kind of variations or distortions in the pulse, significantly affect the output of the Rogowski coil. To avoid any loss of high frequency components present in the practical pulse, the simulated model of Rogowski coil is assigned to measure the same practical primary current. This ensures the correct assessment for evaluation of the identified parameters of the coil to create an accurate model. The measured parameters (Table 2) of the coil are used for simulation of model. The current carrying line used in the experimental setup is shown as test line in the simulation.

Two types of ATP models are shown in Figure 13(b) and Figure 13(c). Block B1 shown in schematic Figure 13(b) represents the PD current pulse source. The coil senses the current through the test circuit. Block B2 represents the time-derivative of the primary current. Block B3 shows the RLC equivalent of Rogowski coil. In the first model, the sensor is electrically connected with the primary line. The arrangement works well as long as the coil circuit does not provide any disturbance to the primary current. However, if any reflections occur within the coil circuit, they may conduct towards the primary (test) line. This phenomenon may affect the characteristics of PD signal. In real practice, the Rogowski coil has no electrical connection with the primary line. Therefore, an improved model is developed in Figure 13(c) in order to isolate the sensor form the line. The Transient Analysis of Control Systems (TACS) shown as block B2 senses the current of the primary line and eliminates the possibility of conducting any reflections from secondary side towards the primary side. In this work, first model has been considered for further analysis and development. The above shown schematics represent the simulation of the Rogowski coil head. Further stages are implemented in Figure 14. 


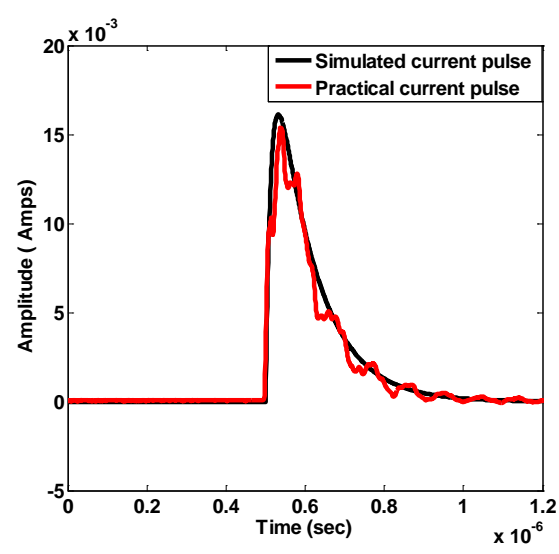

(a)

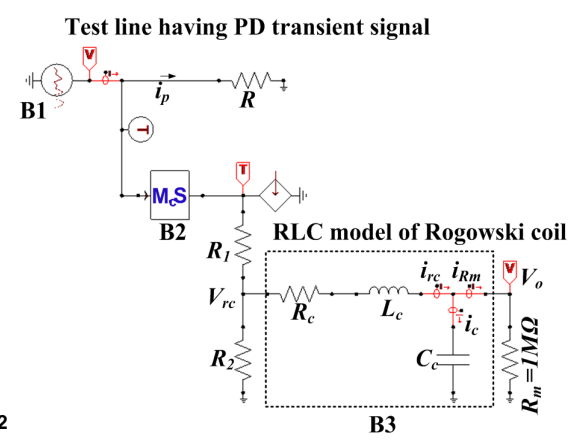

(b)

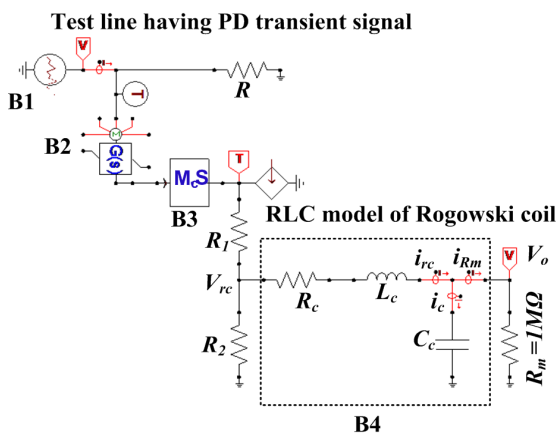

(c)

Figure 13. (a) Comparison of mathematical and practical current pulses; (b) ATP simulation model of Rogowski coil head using RLC equivalent circuit.

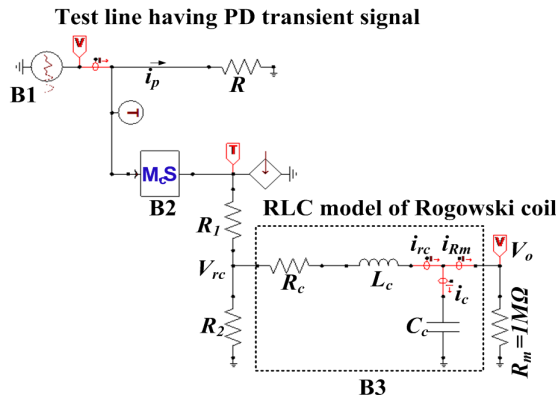

(a)



(d)

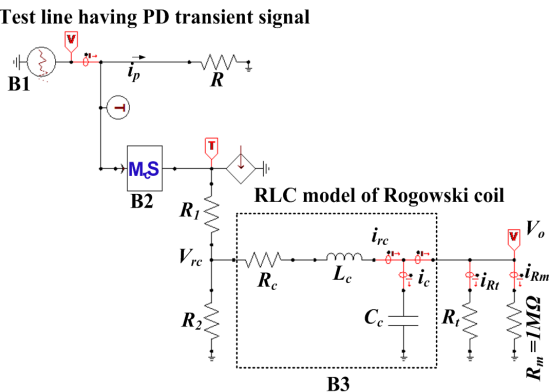

(b)

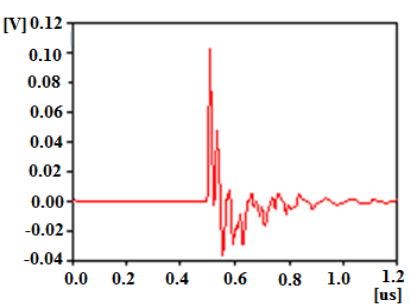

(e)

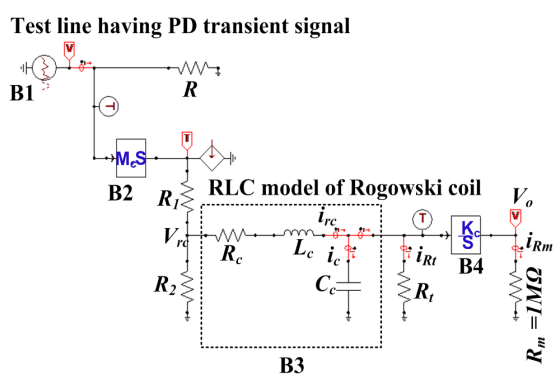

(c)

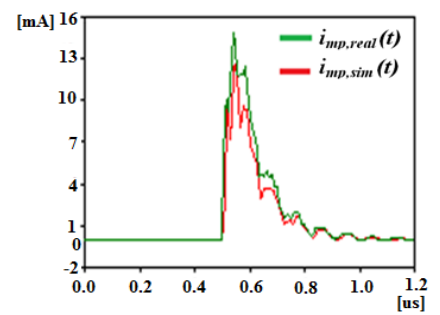

(f)

Figure 14. (a), (b), and (c) shows stage 1, stage 2, and stage 3 respectively; (d), (e), and (f) shows the output of Rogowski coil at stage 1 , stage 2 , and stage 3 respectively. $i_{p m \text {,real }}(t)$ is primary current while $i_{m p \text {,sim }}(t)$ is the measured current of simulated coil.

The output voltage $V_{o}(t)$ is measured with the voltage probe. A comparison is carried out considering the time domain performance and FFT analysis of the measured and simulated outputs. The comparison has proven a good match. This not only ensures the correct identification of the parameters of Rogowski coil but also validates the simulated model of the coil head with enough accuracy for further analysis. It must be emphasized that the sampling rate of the simulation setup must be set as same as that of the practical measurements in order to have a valid comparison between the signals of real and simulated model.

The stage-wise simulated models and their outputs are shown in Figure 14. Extending the initial model, Figure 14 shows the models including coil head added with terminating resistor, and complete Rogowski coil unit including coil head, terminating resistance, integration, and calibration. The measured waveforms of the simulated model have been shown in Figures 14(d)-(f), under their respective stage of model. The performance of the simulated models has a good match with the practically measured response. A comparison of the measured and simulated output response of the coil models is shown in Figure 14(f). The waveform and amplitude of the measured current nicely matches and validates the designed model of Rogowski coil PD measurements. 


\section{Conclusions}

For employing successful on-line monitoring of the distribution lines using PD monitoring, the tasks to be carried out include PD detection, localization, and measurement which consequently provide the information about the presence of PD activity, where the deterioration is happening and extent of the damage that has been done. Such information determines the execution of the required (repair, replacement or standby) tasks. This paper presents design of high-frequency Rogowski coil for PD diagnostics. Rogowski coil is designed in four cascaded stages. First stage is the design of Rogowski coil head, which is used to sense the primary current as an induction sensor. In this form, Rogowski coil can be used to detect the presence and polarity of PD signals which are sufficient to detect and localize the PD fault within the electrical components [17]-[19]. PD measurement task requires quantification of the discharged phenomenon which needs the information of the wave shape of actual PD signals. The area of the PD transient waveform is used to calculate the amount of charge released during PD event and hence the extent of PD defect can be estimated. For this purpose the implementations of the second, third, and fourth stages are necessary.

Rogowski coil head along with damping components is analogue while integration, calibration, and data recording are done by the digital component (DSO). Nowadays many of the DSOs have numerical integration function as a built-in feature and make the integration possible without any additional complex circuits and cost. Similarly calibration constant can be applied numerically. Due to limitations of cost, weight, size, and vast scope of applications for measurements, this device (DSO) is generally used for laboratory or certain onsite applications. However the applications where a large number of coil sensors are integrated into the network, the digital part of the Rogowski coil sensor can be developed using on-chip components and analog-to-digital converters with required sampling rate having programmable signal processor units and data logging and storage functions.

For creating more complex monitoring systems, the ATP-EMTP simulation software has been shown to provide good results in the transient analysis. The design input is simplified by ATP-EMTP as it provides graphical and mouse-driven preprocessor, where the user can construct a wide range of power system circuits. A variety of line models (taking into account the high frequency effects) can be developed by input of the geometrical design parameter which provides an opportunity to further develop and/or analyze diagnostic techniques for PDs travelling over the power lines.

\section{Acknowledgements}

The research was supported by Smart Control Architecture for Smart Grids—SAGA project at Aalto University. The authors highly appreciate the valuable and interesting discussions with Dr. Petri Hyvönen, Aalto University.

\section{References}

[1] Mahmoudi, M., Barkany, A. and Khalfi, A. (2014) A Maintenance Optimization Policy for an Electric Power Distribution System: Case of the HV/MV Substations. Engineering, 6, 236-253. http://dx.doi.org/10.4236/eng.2014.65028

[2] Electro Technical Commission (IEC) 60270, High-Voltage Test Techniques.

[3] Moore, P.J., Portugues, I.E. and Glover, I.A. (2005) Radiometric Location of Partial Discharge Sources on Energized High-Voltage Plant. IEEE Transactions on Power Delivery, 20, 2264-2272. http://dx.doi.org/10.1109/TPWRD.2004.843397

[4] Muhr, M., Strehl, T., Gulski, E., Feser, K., Gockenbach, E., Hauschild, W. and Lemke, E. (2006) Sensors and Sensing Used for Non-Conventional PD Detection. CIGRE, Paris, 1-7.

[5] Schwarz, R., Muhr, M. and Pack, S. (2005) Evaluation of Partial Discharge Impulses with Optical and Conventional Detection Systems. Proceedings of the $24^{\text {th }}$ International Symposium on High Voltage Engineering, Beijing.

[6] Lundgaard, L.E. (1992) Partial Discharge. XIV. Acoustic Partial Discharge Detection-Practical Application. IEEE Electrical Insulation Magazine, 8, 34-43. http://dx.doi.org/10.1109/57.156943

[7] Zhao, X.L., Asada, T., Odendaal, W.G. and Van Wyk, J.D. (2003) An Overview of Integratable Current Sensor Technologies. Industry Applications Conference, 38th IAS Annual Meeting, 2, 1251-1258.

[8] Robles, G., Argueso, M., Sanz, J., Giannetti, R. and Tellini, B. (2007) Identification of Parameters in a Rogowski Coil Used for the Measurement of Partial Discharges. IEEE Instrumentation and Measurement Technology Conference (IMTC) Proceedings, Warsaw, 1-3 May 2007, 1-4.

[9] Jalebi, A. and McMahon, R. (2007) High-Performance Low-Cost Rogowski Transducers and Accompanying Circuitry. 
IEEE Transactions on Instrumentation and Measurement, 3, 354-358.

[10] Poncelas, O., Rosero, J.A., Cusido, J., Ortega, J.A. and Romeral, L. (2008) Design and Application of Rogowski Coil Current Sensor without Integrator for Fault Detection in Induction Motors. IEEE International Symposium on Industrial Electronics, Cambridge, 30 June-2 July 2008, 558-563.

[11] Zhu, J., Yang, L., Jia, J. and Zhang, Q. (2005) The Design of Rogowski Coil with Wide Band Using for Partial Discharge Measurements. Proceeding of International Symposium on Electrical Insulating Materials, 5-9 June 2005, 518521.

[12] Kojovic, L. (1997) Rogowski Coils Suit Relay Protection and Measurement. IEEE Computer Applications in Power, 10, 47-52. http://dx.doi.org/10.1109/67.595293

[13] Knight, D.W. (2010) The Self-Resonance and Self-Capacitance of Solenoid Coils. Version 0.01 (Provisional).

[14] Shafiq, M., Kütt, L., Lehtonen, M., Nieminen, T. and Hashmi, M. (2013) Parameters Identification and Modeling of High Frequency Current Transducer for Partial Discharge Measurements. IEEE Sensors Journal, 13, 1081-1091. http://dx.doi.org/10.1109/JSEN.2012.2227712

[15] Shafiq, M., Lethonen, M., Kutt, L., Hussain, G.A. and Hashmi, M. (2013) Effect of Terminating Resistance on High Frequency Behavior of Rogowski Coil for Transient Measurements. Journal of Electronics and Electrical Engineering (Elektronika Ir Elektrotechnika), 19, 22-28.

[16] Lance, D.S. (2004) An Accelerometer Based Approach to Measuring Displacement of a Vehicle Body. University of Michiga, Dearborn.

[17] Shafiq, M., Kütt, L., Isa, M., Hashmi, M. and Lehtonen, M. (2012) Directional Calibration of Rogowski Coil for Localization of Partial Discharges in Smart Distribution Networks. International Review of Electrical Engineering, 7, 58815890.

[18] Shafiq, M., Lehtonen, M., Isa, M. and Kutt, L. (2013) Online Partial Discharge Diagnostics in Medium Voltage Branched Cable Networks. Proceedings of IEEE International Conference on Power Engineering, Energy and Electrical Drives (POWERENG 2013), Istanbul, 13-17 May 2013, 246-251. http://dx.doi.org/10.1109/PowerEng.2013.6635614

[19] Kane, C. and Golubev, A. (2010) Field Results of Monitoring Partial Discharges on In-Service Large Power Transformers. Proceedings of the Conference Record of the IEEE International Symposium on Electrical Insulation, San Diego, 6-9 June 2010, 1-8. 
Scientific Research Publishing (SCIRP) is one of the largest Open Access journal publishers. It is currently publishing more than 200 open access, online, peer-reviewed journals covering a wide range of academic disciplines. SCIRP serves the worldwide academic communities and contributes to the progress and application of science with its publication.

Other selected journals from SCIRP are listed as below. Submit your manuscript to us via either submit@scirp.org or Online Submission Portal.
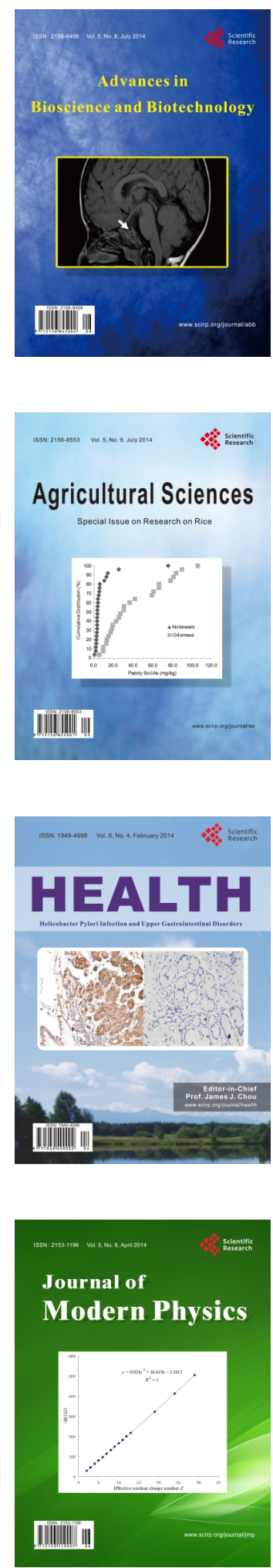
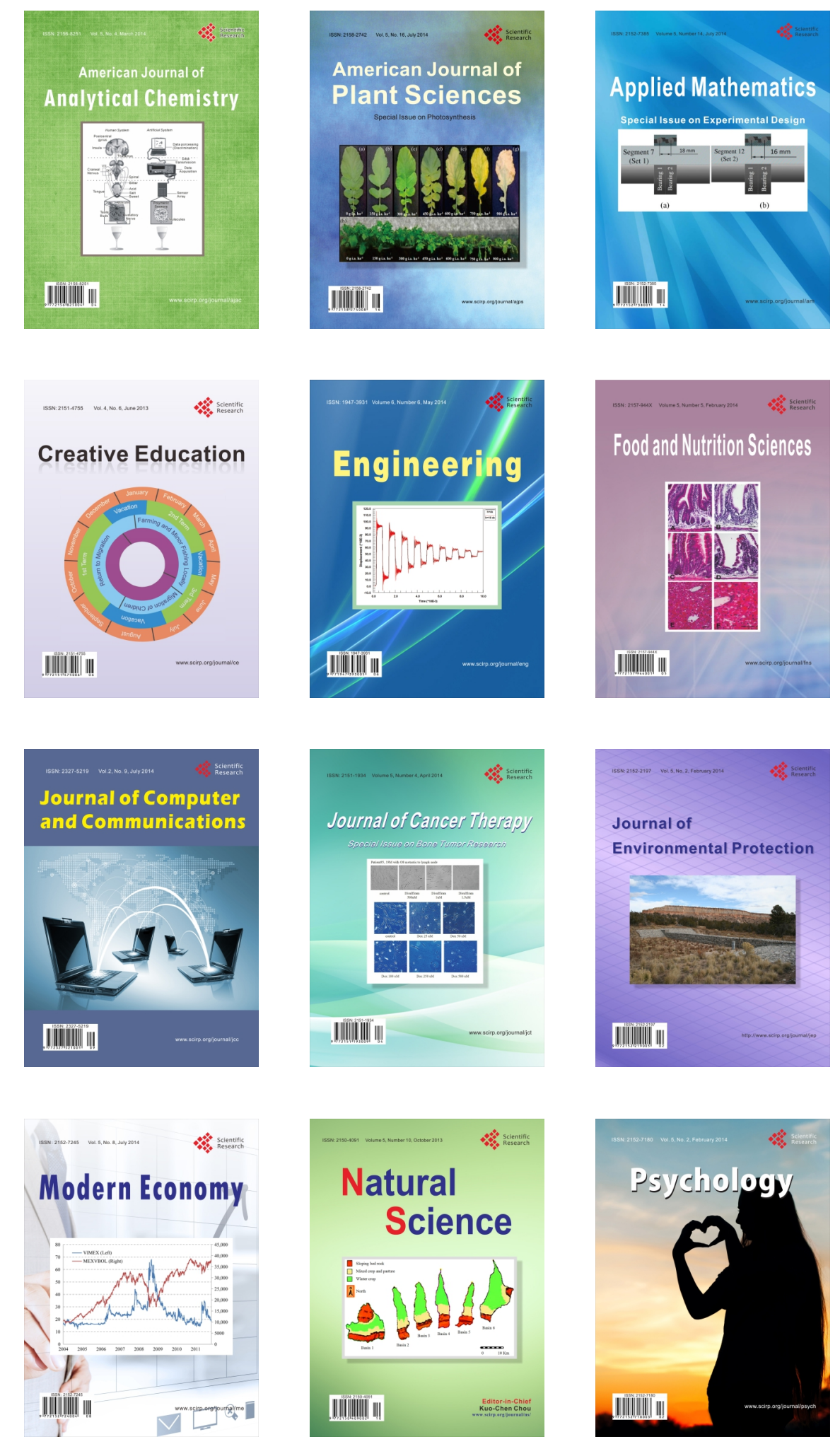